TRANSACTIONS OF THE

AMERICAN MATHEMATICAL SOCIETY

Volume 358, Number 12, December 2006, Pages 5485-5500

S 0002-9947(06)03891-8

Article electronically published on July 20, 2006

\title{
ON A CLASS OF SPECIAL LINEAR SYSTEMS OF $\mathbb{P}^{3}$
}

\author{
ANTONIO LAFACE AND LUCA UGAGLIA
}

\begin{abstract}
In this paper we deal with linear systems of $\mathbb{P}^{3}$ through fat points. We consider the behavior of these systems under a cubic Cremona transformation that allows us to produce a class of special systems which we conjecture to be the only ones.
\end{abstract}

\section{INTRODUCTION}

Let us take the projective space $\mathbb{P}^{n}$ and let us consider the linear system of hypersurfaces of degree $d$ having some points of fixed multiplicity. The virtual dimension of such systems is the dimension of the system of degree $d$ polynomials minus the conditions imposed by the multiple points, and the expected dimension is the maximum between the virtual one and -1 . The systems whose dimension is bigger than the expected one are called special systems.

There exists a conjecture due to Harbourne (see [7]) and Hirschowitz (see [9]), characterizing special linear systems on $\mathbb{P}^{2}$, which has been proved in some special cases (see [2, 3, 12, 11]).

In this paper we describe a class of special linear systems on $\mathbb{P}^{3}$. The main tool will be the cubic Cremona transformation (2.1) which allows us to transform a linear system into another one. The dimension of the two systems is the same, while the virtual one may be different. This is a new phenomenon which does not occur in $\mathbb{P}^{2}$. In Proposition 2.3 we give a formula expressing the difference between these virtual dimensions, and in particular we prove that if a transformation decreases the degree of a system, then it does not decrease its virtual dimension (Corollary 2.4). We will say that a system for which it is no longer possible to decrease the degree using one of these transformations is in standard form. Starting from a special system in standard form it is possible to construct infinitely many special systems by applying a sequence of cubic Cremona transformations. In this paper we describe two types of special systems in standard form, and we conjecture that they produce all the possible special cases.

The paper is organized as follows: in the first section we recall some definitions and notations. In Section 2 we give a description of a cubic Cremona transformation of $\mathbb{P}^{3}$ and its action on linear systems, while Section 3 deals with the resolution of the indeterminacy of this transformation. In Section 4 we state the conjecture and we give a procedure for evaluating the dimension of a system, and in the next section we give some motivations. Section 6 deals with special homogeneous linear

Received by the editors December 8, 2003 and, in revised form, November 2, 2004.

2000 Mathematics Subject Classification. Primary 14C20.

Key words and phrases. Linear systems, fat points.

(C)2006 American Mathematical Society Reverts to public domain 28 years from publication 
systems according to the conjecture, and finally Section 7 provides some examples related to the procedure.

\section{Preliminaries}

We start by fixing some definitions and notations.

Definition 1.1. Given a sheaf $\mathcal{F}=\mathcal{O}_{\mathbb{P}^{3}}(d) \otimes \mathcal{I}_{V}$, where $\mathcal{I}_{V}$ is the ideal sheaf of a subscheme $V \subset \mathbb{P}^{3}$, we denote by $v(\mathcal{F})$ its virtual dimension, defined as

$$
v(\mathcal{F})=\chi(\mathcal{F})-1 .
$$

By $\mathcal{L}=\mathcal{L}_{3}\left(d, m_{1}, \ldots, m_{r}\right)$ we will denote the linear system associated to the sheaf $\mathcal{O}_{\mathbb{P}^{3}}(d) \otimes \mathcal{I}_{Z}$, where $Z=\sum m_{i} p_{i}$ is a zero-dimensional scheme of fat points in general position. If no confusion arises, by abuse of notation we will use the same letter $\mathcal{L}$ to also denote the sheaf. From the cohomology exact sequence associated to

$$
0 \longrightarrow \mathcal{L} \longrightarrow \mathcal{O}_{\mathbb{P}^{3}}(d) \longrightarrow \mathcal{O}_{Z} \longrightarrow 0,
$$

we obtain that $h^{i}(\mathcal{L})=0$ for $i=2,3$. Therefore we deduce that $v(\mathcal{L})=h^{0}\left(\mathcal{O}_{\mathbb{P}^{3}}(d)\right)$ $h^{0}\left(\mathcal{O}_{z}\right)-1$, which may also be written as

$$
v(\mathcal{L})=\left(\begin{array}{c}
d+3 \\
3
\end{array}\right)-\sum_{i=1}^{r}\left(\begin{array}{c}
m_{i}+2 \\
3
\end{array}\right)-1 .
$$

Let us denote by $e(\mathcal{L})=\max (v(\mathcal{L}),-1)$ the expected dimension of $\mathcal{L}$.

Definition 1.2. A non-empty linear system $\mathcal{L}$ is special if its expected dimension is strictly smaller than the effective one or, equivalently, if $h^{1}(\mathcal{L}) \neq 0$.

Let $(X, \pi)$ be the blowing-up of $\mathbb{P}^{3}$ along $\left\{p_{1}, \ldots, p_{r}\right\}$; by abuse of notation we will denote by $\mathcal{L}$ the linear system associated to $L=d H-\sum m_{i} E_{i}$, where $H$ is the pull-back of a hyperplane of $\mathbb{P}^{3}$ and $E_{i}=\pi^{-1}\left(p_{i}\right)$. Let $\left\langle H, E_{1}, \ldots, E_{r}\right\rangle$ and $\left\langle h, e_{1}, \ldots, e_{r}\right\rangle$ be two bases for the Chow groups $A^{1}(X)$ and $A^{2}(X)$, respectively, where $h$ is the pull-back of a line and $e_{i}$ is the class of a line in $E_{i}$. The intersection matrix, with respect to these two bases, is diagonal with the first element equal to 1 and the others equal to -1 (since $E_{i} e_{i}=-1$ ).

Given a curve $C \subset \mathbb{P}^{3}$, by abuse of notation we will denote by $\mathcal{L} C$ the intersection product of their strict transforms in $X$. We will write $C \in \ell_{3}\left(\delta, \mu_{1}, \ldots, \mu_{r}\right)$ to denote a curve of degree $\delta$ with multiplicity $\mu_{i}$ at $p_{i}$. In this way, the intersection is given by the formula

$$
\mathcal{L} C=d \delta-\sum_{i=1}^{r} \mu_{i} m_{i}
$$

We recall the Riemann-Roch formula for a divisor $L$ on a smooth threefold $X$ :

$$
\chi(L)=\frac{L\left(L-K_{X}\right)\left(2 L-K_{X}\right)+c_{2}(X) L}{12}+\chi\left(\mathcal{O}_{X}\right) .
$$

If the linear system associated to $L$ can be written as $|L|=F+|M|$, where $F$ is a fixed divisor of $|L|$ and $|M|$ is the residual system, then the above formula implies

$$
v(L)=v(M)+v(F)+\frac{F M\left(L-K_{X}\right)}{2} .
$$




\section{Cubic Cremona transformations and linear systems}

In this section we focus our attention on a class of cubic Cremona transformations of $\mathbb{P}^{3}$. Consider the system $\mathcal{L}_{3}\left(3,2^{4}\right)$. By putting the four double points in the fundamental ones, the associated rational map is given by

$$
\mathrm{Cr}:\left(x_{0}: x_{1}: x_{2}: x_{3}\right) \rightarrow\left(x_{0}^{-1}: x_{1}^{-1}: x_{2}^{-1}: x_{3}^{-1}\right)
$$

The birational map (2.1) induces an action on the Picard group of $X$ which can be described in the following way.

Proposition 2.1. The action of transformation (2.1) on $\mathcal{L}=\mathcal{L}_{3}\left(d, m_{1}, \ldots, m_{r}\right)$ is given by

$$
\operatorname{Cr}(\mathcal{L})=\mathcal{L}_{3}\left(d+k, m_{1}+k, \ldots, m_{4}+k, m_{5}, \ldots, m_{r}\right)
$$

where $k=2 d-\sum_{i=1}^{4} m_{i}$.

Proof. Since the transformation (2.1) is an isomorphism outside the base locus, we can reduce to the case $r=4$. The exponents of monomials which generate $\mathcal{L}$ (i.e. monomials of degree $d$ in $x_{1}, \ldots, x_{4}$ with multiplicity $m_{i}$ at $p_{i}$ for $i=1, \ldots, 4$ ) can be represented by the points of the truncated lattice tetrahedron

$$
T:=\left\{\left(a_{1}, \ldots, a_{4}\right) \in \mathbb{Z}^{4} \mid 0 \leq a_{i} \leq d-m_{i} \text { and } \sum_{i=1}^{4} a_{i}=d\right\} .
$$

In the same way $T^{\prime}$ corresponds to $\mathcal{L}_{3}\left(d+k, m_{1}+k, \ldots, m_{4}+k\right)$. Observe that

$$
\operatorname{Cr}\left(\prod_{i=1}^{4} x_{i}{ }^{a_{i}}\right)=\prod_{i=1}^{4} x_{i}{ }^{d-a_{i}}
$$

where the right term may be written as

$$
\left(x_{1}^{m_{1}} \cdots x_{4}^{m_{4}}\right) \prod_{i=1}^{4} x_{i}^{d-a_{i}-m_{i}} .
$$

This may be summarized by saying that the transformation (2.1) induces the map $f\left(a_{1}, \ldots, a_{4}\right)=\left(d-a_{1}-m_{1}, \ldots, d-a_{4}-m_{4}\right)$ from $\mathbb{Z}^{4}$ to $\mathbb{Z}^{4}$. We want to prove that $f$ is a bijection between $T$ and $T^{\prime}$. First of all observe that if $\left(a_{1}, \ldots, a_{4}\right) \in T$, then $0 \leq d-a_{i}-m_{i} \leq(d+k)-\left(m_{i}+k\right)$ and $\sum_{i=1}^{4}\left(d-a_{i}-m_{i}\right)=d+k$, which implies that $f(T) \subseteq T^{\prime}$. In the same way it is possible to prove that $f\left(T^{\prime}\right) \subseteq T$ and, since $f^{2}=\mathbf{1}_{\mathbb{Z}^{4}}$, we get the claim.

Observe that $\operatorname{dim} \operatorname{Cr}(\mathcal{L})=\operatorname{dim} \mathcal{L}$, but in general the virtual dimensions of the two systems may be different.

Lemma 2.2. Suppose that $2 d-m_{1}-m_{2}-m_{3}<0$. Then $\operatorname{Bs} \mathcal{L}_{3}\left(d, m_{1}, m_{2}, m_{3}\right)$ contains the plane through the three points.

Proof. Consider $T$ as before (with $m_{4}=0$ ). Then we have the inequality $a_{1}+a_{2}+$ $a_{3} \leq 3 d-m_{1}-m_{2}-m_{3}<d$. This implies that each monomial contains $x_{4}$, which gives the fixed plane. 
Proposition 2.3. Let $\mathcal{L}=\mathcal{L}_{3}\left(d, m_{1}, \ldots, m_{r}\right)$ be a linear system such that $2 d \geq$ $m_{i}+m_{j}+m_{k}$ for any choice of $\{i, j, k\} \subset\{1,2,3,4\}$. Then

$$
v(\operatorname{Cr}(\mathcal{L}))-v(\mathcal{L})=\sum_{t_{i j} \geq 2}\left(\begin{array}{c}
1+t_{i j} \\
3
\end{array}\right)-\sum_{t_{i j} \leq-2}\left(\begin{array}{c}
1-t_{i j} \\
3
\end{array}\right),
$$

where $t_{i j}=m_{i}+m_{j}-d$.

Proof. We can reduce to the case $\mathcal{L}=\mathcal{L}_{3}\left(d, m_{1}, \ldots, m_{4}\right)$, since the contribution of the other points is 0 in equation (2.4). In this case, $\operatorname{dim} \mathcal{L}=\# T-1$, where $T$ is the set defined in (2.3). In order to evaluate this number, consider the sets

$$
\begin{aligned}
\Delta & :=\left\{\left(a_{1}, \ldots, a_{4}\right) \in \mathbb{Z}_{\geq 0}^{4} \mid \sum_{i=1}^{4} a_{i}=d\right\}, \\
\Delta_{i} & :=\left\{\left(a_{1}, \ldots, a_{4}\right) \in \Delta \mid a_{i} \geq d-m_{i}+1\right\},
\end{aligned}
$$

and observe that $T=\Delta \backslash \bigcup_{i=1}^{4} \Delta_{i}$. By the inclusion-exclusion principle,

$$
\# T=\# \Delta-\sum_{i} \# \Delta_{i}+\sum_{i<j} \#\left(\Delta_{i} \cap \Delta_{j}\right)-\sum_{i<j<k} \#\left(\Delta_{i} \cap \Delta_{j} \cap \Delta_{k}\right) .
$$

The virtual dimension $v(\mathcal{L})$ is one less than the difference of the first two terms on the right side of the equation above. A point $\left(a_{1}, \ldots, a_{4}\right)$ belongs to the intersection of the first three $\Delta_{i}$ 's if $a_{i}>d-m_{i}$ for $i=1,2,3$; summing up these inequalities one obtains $d-a_{4}>3 d-m_{1}-m_{2}-m_{3}$ which implies that $2 d-m_{1}-m_{2}-m_{3}<0$, a contradiction to our assumption. The same argument holds for any other triple of points, so this gives the following formula:

$$
\operatorname{dim} \mathcal{L}=v(\mathcal{L})+\sum_{i<j} \#\left(\Delta_{i} \cap \Delta_{j}\right)
$$

The set $\Delta_{1} \cap \Delta_{2}$ is in one-to-one correspondence with the tetrahedron

$$
\Delta_{12}:=\left\{\left(b_{1}, \ldots, b_{4}\right) \in \mathbb{Z}_{\geq 0}^{4} \mid \sum_{i=1}^{4} b_{i}=m_{1}+m_{2}-d-2\right\},
$$

where $b_{i}=a_{i}-d-1+m_{i}$ for $i=1,2$ and $b_{i}=a_{i}$ for $i=3,4$. The same holds for the other intersections and, since $m_{i}+m_{j}-d-2=t_{i j}-2$, we have $\#\left(\Delta_{i} \cap \Delta_{j}\right)=\left(\begin{array}{c}t_{i j}+1 \\ 3\end{array}\right)$. The equality $\operatorname{dim} \mathcal{L}=\operatorname{dim} \operatorname{Cr}(\mathcal{L})$ implies that

$$
v(\operatorname{Cr}(\mathcal{L}))-v(\mathcal{L})=\sum_{t_{i j} \geq 2}\left(\begin{array}{c}
t_{i j}+1 \\
3
\end{array}\right)-\sum_{t_{i j}^{\prime} \geq 2}\left(\begin{array}{c}
t_{i j}^{\prime}+1 \\
3
\end{array}\right),
$$

where $t_{i j}^{\prime}$ is defined in the same way as $t_{i j}$ for the system $\operatorname{Cr}(\mathcal{L})$. This implies that $t_{12}^{\prime}=\left(m_{1}+k\right)+\left(m_{2}+k\right)-(d+k)=t_{12}+k=-t_{34}$, and an analogous equality holds for each $t_{i j}^{\prime}$.

Corollary 2.4. Under the assumptions of Proposition 2.3, if the degree of $\operatorname{Cr}(\mathcal{L})$ is less than that of $\mathcal{L}$, then $v(\operatorname{Cr}(\mathcal{L})) \geq v(\mathcal{L})$.

Proof. The difference between the degree of $\operatorname{Cr}(\mathcal{L})$ and that of $\mathcal{L}$ is equal to $k=$ $2 d-\sum_{i=1}^{4} m_{i}$. From $2 d<\sum_{i=1}^{4} m_{i}$ we deduce that, if $t_{12} \geq 2$, then $d-m_{3}-m_{4}<$ $m_{1}+m_{2}-d$ which is equivalent to $-t_{34}<t_{12}$. The same holds for each $t_{i j}$ such that $t_{i j} \geq 2$, hence the right side of equation (2.4) is non-negative. 


\section{The IsOMORPHISMS of $\mathrm{A}^{i}(Y)$ \\ INDUCED BY THE CUBIC TRANSFORMATION}

The resolution of the indeterminacy of map (2.1) may be expressed by the following diagram:

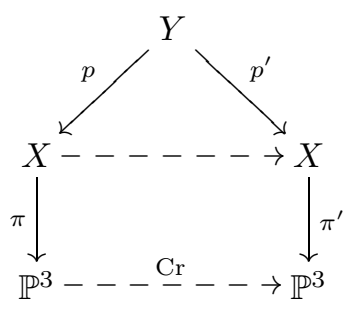

Here $\pi$ is the blowing-up of $\mathbb{P}^{3}$ along the points $p_{1}, \ldots, p_{4}$, and $p$ is the blowing-up of $X$ along the strict transforms of the lines $l_{i j}$ through $p_{i}$ and $p_{j}$. The Picard group of $X$ is generated by $\left\langle H, E_{1}, \ldots, E_{4}\right\rangle$, where $E_{i}$ is the exceptional divisor corresponding to $p_{i}$. Let us denote $F_{i}=p^{*} E_{i}$ (this means that $F_{i}$ is the blowing-up of a plane through three points), and let $F_{i j}$ be the exceptional divisor corresponding to the blowing-up of the strict transform of $l_{i j}$. The divisor $F_{i j}$ is a quadric, and we will denote by $f_{i j}^{v}$ its "vertical" ruling, i.e. the one given by the lines of the blowing-up, and by $f_{i j}^{h}$ the other one (the "horizontal"). The morphism $p^{\prime}$ contracts the quadrics $F_{i j}$ along the horizontal rulings, while the divisors contracted by $\pi^{\prime}$ are the four fundamental planes. A base for $\operatorname{Pic}(Y)$ is given by $\left\langle H, F_{1}, \ldots, F_{4}, F_{12}, \ldots, F_{34}\right\rangle$, where, with abuse of notation, we write $H$ instead of $p^{*} H$. Let $H_{1}=H-F_{2}-$ $F_{3}-F_{4}-F_{23}-F_{24}-F_{34}$ be the strict transform of the plane of $\mathbb{P}^{3}$ through the points $p_{2}, p_{3}, p_{4}$ and the three lines of the triangle. In the same way we define $H_{i}$ for $i=2,3,4$. The involution $\mathrm{Cr}: \operatorname{Pic}(Y) \longrightarrow \operatorname{Pic}(Y)$ induced by (2.1) is given by

$$
\operatorname{Cr}\left(F_{i}\right)=H_{i}, \quad \operatorname{Cr}\left(F_{i j}\right)=F_{h k},
$$

where $\{h, k\}=\{1,2,3,4\} \backslash\{i, j\}$, which gives

$$
\operatorname{Cr}(H)=3 H-\sum_{i} 2 F_{i}-\sum_{i<j} F_{i j} .
$$

Proposition 3.1. Let $Z$ be the 0-dimensional scheme of four points $p_{1}, \ldots, p_{4}$ with multiplicities $m_{1}, \ldots, m_{4}$ and let $W$ be the 1-dimensional scheme of the lines $\left\langle p_{i}, p_{j}\right\rangle$ with multiplicities $n_{i j}$. The map (2.1) transforms the sheaf $\mathcal{F}=\mathcal{O}_{\mathbb{P}^{3}}(d) \otimes \mathcal{I}_{Z} \otimes \mathcal{I}_{W}$ into $\mathcal{F}^{\prime}=\mathcal{O}_{\mathbb{P}^{3}}(d+s) \otimes \mathcal{I}_{Z^{\prime}} \otimes \mathcal{I}_{W^{\prime}}$, where $s=2 d-\sum m_{i}, m_{i}^{\prime}=m_{i}+s$ and $n_{i j}^{\prime}=d-m_{i}-m_{j}+n_{h k}$.

Proof. Let us take the pull-back of $\mathcal{F}$ on $Y$ :

$$
p^{*} \pi^{*} \mathcal{F}=d H-\sum_{i} m_{i} F_{i}-\sum_{i<j} n_{i j} F_{i j} .
$$

By (3.2) and (3.3) its image under the map $\mathrm{Cr}$ is

$$
\operatorname{Cr}\left(p^{*} \pi^{*} \mathcal{F}\right)=(d+s) H-\sum_{i}\left(m_{i}+s\right) F_{i}-\sum_{i<j}\left(d-m_{i}-m_{j}+n_{h k}\right) F_{i j},
$$

which finishes the proof. 
To each linear system $\mathcal{L}=\mathcal{L}_{3}\left(d, m_{1}, \ldots, m_{4}\right)$ we associate a 1 -cycle defined as

$$
\Gamma(\mathcal{L}):=\sum_{t_{i j} \geq 1} t_{i j} l_{i j}
$$

where $t_{i j}=m_{i}+m_{j}-d$ and $l_{i j}$ is the line through $p_{i}$ and $p_{j}$. Observe that by definition $H^{0}\left(\mathcal{L} \otimes \mathcal{I}_{\Gamma(\mathcal{L})}\right)=H^{0}(\mathcal{L})$, since each line $l_{i j} \in \Gamma(\mathcal{L})$ is contained in the base locus of $\mathcal{L}$ with multiplicity at least $t_{i j}$.

Proposition 3.2. With the preceding notations we have

$$
\chi\left(\mathcal{L} \otimes \mathcal{I}_{\Gamma(\mathcal{L})}\right)=\chi(\mathcal{L})+\sum_{t_{i j} \geq 2}\left(\begin{array}{c}
t_{i j}+1 \\
3
\end{array}\right) .
$$

Proof. We recall that by abuse of notation we denote by $\mathcal{L}$ the linear system on $X$ associated to $d H-\sum m_{i} E_{i}$. Consider the pull-back of $\mathcal{L} \otimes \mathcal{I}_{\Gamma(\mathcal{L})}$ on $Y$. In what follows we will concentrate on one of the six lines, and in order to simplify the notations we will omit the indices. Let $l \in \mathbf{A}^{2}(X)$ be the strict transform of the line, then $N_{l \mid X} \cong \mathcal{O}_{\mathbb{P}^{1}}(-1) \oplus \mathcal{O}_{\mathbb{P}^{1}}(-1)$. From the evaluation of the tautological line bundle associated to the blowing-up of $l$ (see [5]), one obtains that $F_{\mid F}=-f^{v}-f^{h}$. The intersection $\mathcal{L} l=-t$ gives $p^{*} \mathcal{L}_{\mid F}=-t f^{v}$. The exact sequence

$$
0 \longrightarrow p^{*} \mathcal{L}-(k+1) F \longrightarrow p^{*} \mathcal{L}-k F \longrightarrow\left(p^{*} \mathcal{L}-k F\right)_{\mid F} \longrightarrow 0
$$

and the preceding formulas imply that $h^{0}\left(\left(p^{*} \mathcal{L}-k F\right)_{\mid F}\right)=h^{0}\left(k f^{h}+(k-t) f^{v}\right)=0$ if and only if $k<t$. Therefore $t F \subseteq \operatorname{Bs}\left(p^{*} \mathcal{L}\right)$ and

$$
\chi\left(p^{*} \mathcal{L}-t F\right)=\chi\left(p^{*} \mathcal{L}\right)-\sum_{k=0}^{t-1} \chi\left(k f^{h}+(k-t) f^{v}\right) .
$$

By the Riemann-Roch theorem on the quadric $F$ we have $\chi\left(k f^{h}+(k-t) f^{v}\right)=$ $(k+1)(k+1-t)$. An easy calculation shows that the last sum of the above equation is equal to $-\left(\begin{array}{c}t+1 \\ 3\end{array}\right)$, hence applying this procedure to each of the $F_{i j}$ one obtains

$$
\chi\left(p^{*} \mathcal{L}-\sum_{t_{i j} \geq 2} t_{i j} F_{i j}\right)=\chi\left(p^{*} \mathcal{L}\right)+\sum_{t_{i j} \geq 2}\left(\begin{array}{c}
t_{i j}+1 \\
3
\end{array}\right) .
$$

The proof follows from the projection formula $p_{*} p^{*} \mathcal{F} \cong \mathcal{F}$ and $R^{i} p_{*} p^{*} \mathcal{F}=0$, with $i \geq 1$, for a blowing-up (see [8]). These properties imply that $H^{i}\left(Y, p^{*} \mathcal{F}\right) \cong$ $H^{i}(X, \mathcal{F})$, where $\mathcal{F}$ is $\mathcal{L}$ or $\mathcal{L} \otimes \mathcal{I}_{\Gamma(\mathcal{L})}$.

Corollary 3.3. Let $\mathcal{L}=\mathcal{L}_{3}\left(d, m_{1}, \ldots, m_{r}\right)$ be a linear system and let $C_{1}, \ldots, C_{n} \in$ $\mathbf{A}^{2}(X)$ be a set of irreducible rational curves such that $N_{C_{i} \mid X} \cong \mathcal{O}_{\mathbb{P}^{1}}(-1) \oplus \mathcal{O}_{\mathbb{P}^{1}}(-1)$ and $\mathcal{L} C_{i}=-t_{i} \leq-2$ for $i=1, \ldots, n$. Then

$$
\operatorname{dim} \mathcal{L}-v(\mathcal{L}) \geq \sum_{i=1}^{n}\left(\begin{array}{c}
t_{i}+1 \\
3
\end{array}\right)-h^{2}\left(\mathcal{L} \otimes \mathcal{I}_{\Gamma}\right),
$$

where $\Gamma:=\sum t_{i} C_{i}$ is the 1-dimensional scheme of the multiple curves.

Proof. Observe that in the proof of Proposition 3.2 the only assumption needed on the curve $l$ is that its normal bundle in $X$ is $\mathcal{O}_{\mathbb{P}^{1}}(-1) \oplus \mathcal{O}_{\mathbb{P}^{1}}(-1)$. Since we are assuming the same for the $C_{i}$, we have that $\chi\left(\mathcal{L} \otimes \mathcal{I}_{\Gamma}\right)=\chi(\mathcal{L})+\sum\left(\begin{array}{c}t_{i}+1 \\ 3\end{array}\right)$. The equalities $h^{0}(\mathcal{L})=h^{0}\left(\mathcal{L} \otimes \mathcal{I}_{\Gamma}\right)$ and $h^{2}(\mathcal{L})=0$ finish the proof. 
Remark 3.4. Let us take a system $\mathcal{L}$, choose four points and consider the associated sheaf $\mathcal{L} \otimes \mathcal{I}_{\Gamma(\mathcal{L})}$. Use Proposition 3.1 to transform this sheaf into $\mathcal{L}^{\prime} \otimes \mathcal{I}_{\Gamma\left(\mathcal{L}^{\prime}\right)}$. The system $\mathcal{L}^{\prime}$ is obtained from $\mathcal{L}$ by applying Proposition 2.1. Observe that $h^{i}\left(\mathcal{L} \otimes \mathcal{I}_{\Gamma(\mathcal{L})}\right)=h^{i}\left(\mathcal{L}^{\prime} \otimes \mathcal{I}_{\Gamma\left(\mathcal{L}^{\prime}\right)}\right)$, since the pull-back of the first system on $Y$ is just the pull-back of the second one obtained by a base change on $\operatorname{Pic}(Y)$. In particular this implies that the virtual dimensions of the two systems are the same. This, together with Proposition 3.2 gives another proof of Proposition 2.3.

Let us now consider the action of transformation (2.1) on the curves of $Y$. A basis for $\mathbf{A}^{2}(Y)$ may be given by $\left\langle h, f_{1}, \ldots, f_{4}, f_{12}, \ldots, f_{34}\right\rangle$, where $h$ is the pullback of the class of a line in $\mathbb{P}^{3}, f_{i}$ is the pull-back of a line of $E_{i}$ and $f_{i j}=f_{i j}^{v}$ is the vertical ruling of $F_{i j}$.

Proposition 3.5. The intersection matrix $M: \mathbf{A}^{1}(Y) \times \mathbf{A}^{2}(Y) \longrightarrow \mathbb{Z}$ with respect to the chosen bases of $\mathbf{A}^{i}(Y)$ is given by

$$
M=\left(\begin{array}{c|c}
1 & 0 \\
\hline 0 & -I
\end{array}\right) .
$$

Proof. By using the projection formula, since $(\pi \circ p)_{*} F_{i}=p_{i}, F_{i}$ has a non-zero intersection only with $f_{i}$ and $F_{i} f_{i}=F_{i \mid F_{i}} f_{i}=-f_{i}^{2}=-1$, where the second equation is due to $F_{i}^{2}=p^{*} E_{i}^{2}=p^{*} N_{E_{i} \mid X}=p^{*}\left(-e_{i}\right)=-f_{i}$. In the same way, since $(\pi \circ p)_{*} F_{i j}=l_{i j}, F_{i j}$ has a non-vanishing intersection only with $f_{i j}$. Let $F$ and $f$ be $F_{i j}$ and $f_{i j}$, respectively; then $F f=F_{\mid F} f=\left(-f^{v}-f^{h}\right) f^{v}=-1$. Finally $H h=1$ since it is the intersection of a plane with a line.

Proposition 3.6. With the same notation as before, we have that $H F_{i j}=f_{i j}$ and $F_{i j}^{2}=-h+f_{i}+f_{j}-2 f_{i j}$.

Proof. As before we will use $F, f$ instead of $F_{i j}, f_{i j}$. The first equality is obvious, since $p_{*} H$ intersects $p_{*} F$ along a point and $f$ is just the vertical fiber of the quadric over that point. Observe that since $F^{2}$ has a non-vanishing intersection only with $H, F_{i}, F_{j}$ and $F$, then by Proposition 3.5 we have $F^{2}=a h+m_{i} f_{i}+m_{j} f_{j}+b f$. The coefficient $a$ is equal to $F^{2} H=F f=-1$. In the same way $m_{i}=-F^{2} F_{i}=-F f=1$ and $b=-F^{3}=-\left(-f^{v}-f^{h}\right)^{2}=-2$.

Proposition 3.7. The action of map (2.1) on $\mathbf{A}^{2}(Y)$ is given by

$$
\operatorname{Cr}(h)=3 h-\sum_{i} f_{i}, \operatorname{Cr}\left(f_{i}\right)=2 h-\sum_{j \neq i} f_{j}, \operatorname{Cr}\left(f_{i j}\right)=h+f_{k s}-f_{k}-f_{s},
$$

where $\{k, s\}=\{1,2,3,4\} \backslash\{i, j\}$.

Proof. Since $h=H^{2}, f_{1}=-F_{1}^{2}$ and $f_{12}=H F_{12}$, we have

$$
\begin{aligned}
\operatorname{Cr}(h) & =\operatorname{Cr}(H)^{2} \\
& =\left(3 H-2 \sum_{i} F_{i}-\sum_{i<j} F_{i j}\right)^{2} \\
& =9 h-4 \sum_{i} f_{i}-\sum_{i<j}\left(h-f_{i}-f_{j}+2 f_{i j}\right)-6 \sum_{i<j} f_{i j}+8 \sum_{i<j} f_{i j} \\
& =3 h-\sum_{i} f_{i},
\end{aligned}
$$




$$
\begin{aligned}
\operatorname{Cr}\left(f_{1}\right)= & -\operatorname{Cr}\left(F_{1}\right)^{2} \\
= & -\left(H-F_{2}-F_{3}-F_{4}-F_{23}-F_{24}-F_{34}\right)^{2} \\
= & -\left(h-f_{2}-f_{3}-f_{4}-3 h+2\left(f_{2}+f_{3}+f_{4}\right)-2\left(f_{23}+f_{24}+f_{34}\right)\right. \\
& \left.-2\left(f_{23}+f_{24}+f_{34}\right)+4\left(f_{23}+f_{24}+f_{34}\right)\right) \\
= & 2 h-f_{2}-f_{3}-f_{4}, \\
\operatorname{Cr}\left(f_{12}\right)= & \operatorname{Cr}(H) \operatorname{Cr}\left(F_{12}\right) \\
= & \left(3 H-2 \sum_{i} F_{i}-\sum_{i<j} F_{i j}\right) F_{34} \\
= & 3 f_{34}-4 f_{34}+h-f_{3}-f_{4}+2 f_{34} \\
= & h+f_{34}-f_{3}-f_{4} .
\end{aligned}
$$

Now, by $\ell_{3}\left(\delta, \mu_{1}, \ldots, \mu_{4}, \beta_{12}, \ldots, \beta_{34}\right)$ we will denote the system of curves of $\mathbb{P}^{3}$ of degree $\delta$ with multiplicities $\mu_{i}$ in the four points and intersecting the line $l_{i j}$ along $\beta_{i j}$ points.

Proposition 3.8. The image of the system $\ell=\ell_{3}\left(\delta, \mu_{1}, \ldots, \mu_{4}, \beta_{12}, \ldots, \beta_{34}\right)$ by transformation (2.1) is $\operatorname{Cr}(\ell):=\ell_{3}\left(\delta^{\prime}, \mu_{1}^{\prime}, \ldots, \mu_{4}^{\prime}, \beta_{12}^{\prime}, \ldots, \beta_{34}^{\prime}\right)$, where

$$
\delta^{\prime}=3 \delta-\sum_{i} 2 \mu_{i}-\sum_{i<j} \beta_{i j}, \quad \mu_{r}^{\prime}=\delta-\sum_{j \neq r} \mu_{j}-\sum_{\{i, j\} \not r} \beta_{i j}, \quad \beta_{i j}^{\prime}=\beta_{k s} .
$$

Proof. The strict transform of a general element of $\ell$ may be written on $Y$ as $\delta h-\sum \mu_{i} f_{i}-\sum \beta_{i j} f_{i j}$. The image of this element by the map $\mathrm{Cr}$ is

$$
\delta\left(3 h-\sum_{i} f_{i}\right)-\sum_{i} \mu_{i}\left(2 h-\sum_{j \neq i} f_{j}\right)-\sum_{i<j} \beta_{i j}\left(h+f_{k s}-f_{k}-f_{s}\right) .
$$

This immediately gives the value of $\delta^{\prime}$ and $\beta_{i j}^{\prime}$. For $\mu_{r}^{\prime}$, observe that $f_{r}$ appears in the first sum with coefficient $-\delta$, in the second with coefficient $\sum_{j \neq r} \mu_{j}$, and in the third sum it appears each time that the index $r \in\{k, s\}$ or, which is the same, each time that $r \notin\{i, j\}$.

If the system $\ell$ has no intersection with any one of the six lines, then we have the following.

Corollary 3.9. The action of transformation (2.1) on $\ell=\ell_{3}\left(\delta, \mu_{1}, \ldots, \mu_{r}\right)$ is given by

$$
\operatorname{Cr}(\ell):=\ell\left(\delta+2 \gamma, \mu_{1}+\gamma, \ldots, \mu_{4}+\gamma, \mu_{5}, \ldots, \mu_{r}\right),
$$

where $\gamma=\delta-\sum_{i=1}^{4} \mu_{i}$.

The action of $\mathrm{Cr}$ on the space of curves may be extended to a linear action on the space of polynomials $\mathbb{C}\left[\delta, \mu_{1}, \ldots, \mu_{r}\right]$. An easy calculation gives

Corollary 3.10. The following polynomials are invariant with respect to transformation (3.5):

$$
2 \delta-\sum_{i=1}^{s} \mu_{i}, \quad \delta^{2}-\sum_{i=1}^{s} 2 \mu_{i}^{2}
$$




\section{Conjecture}

In this section we state a conjecture which allows us to give a procedure for computing the dimension of a linear system $\mathcal{L}=\mathcal{L}_{3}\left(d, m_{1}, \ldots, m_{r}\right)$. First of all, since birational transformations do not change the effective dimension of $\mathcal{L}$, we can perform Cremona transformations until we get a system in standard form. If at some step we get a system $\mathcal{L}^{k}$ with a negative multiplicity $-\alpha_{i}$ at $p_{i}$, then the system contains $\alpha_{i} E_{i}$ in its base locus. We remark that if this is the case, then there also exists a fixed component contained $\alpha_{i}$ times in the base locus of the starting system $\mathcal{L}$. This is the image of $E_{i}$ by the sequence of Cremona transformations sending $\mathcal{L}^{k}$ back to $\mathcal{L}$. This component can be removed without changing the dimension of $\mathcal{L}$. In particular we can remove $\alpha_{i} E_{i}$ from $\mathcal{L}^{k}$ and keep performing Cremona if possible. That way we can reduce the problem to the study of the dimension of linear systems in standard form. In this direction let us state the following.

Conjecture 4.1. A linear system $\mathcal{L}=\mathcal{L}_{3}\left(d, m_{1}, \ldots, m_{r}\right)$ in standard form is special if and only if one of the following holds:

(i) there exists a quadric $Q=\mathcal{L}_{3}\left(2,1^{9}\right)$ such that $Q(\mathcal{L}-Q)(\mathcal{L}-K)<0$;

(ii) there exists a line $\ell=\ell_{3}\left(1,1^{2}\right)$ such that $\mathcal{L} \ell \leq-2$.

We remark that if condition (i) holds, then by (1.2) $v(\mathcal{L})<v(\mathcal{L}-Q)$, while $\operatorname{dim} \mathcal{L} \geq \operatorname{dim}(\mathcal{L}-Q)$, which means that $\mathcal{L}$ is special. In order to simplify the procedure we are going to prove that, under an extra assumption, condition (i) implies that the quadric $Q$ is contained in the base locus of $\mathcal{L}$.

Lemma 4.2. Let us suppose that the Harbourne-Hirschowitz Conjecture holds for linear systems on $\mathbb{P}^{2}$ with 10 fixed points. If $\mathcal{L}=\mathcal{L}_{3}\left(d, m_{1}, \ldots, m_{r}\right)$ is a system in standard form and such that $Q(\mathcal{L}-Q)(\mathcal{L}-K)<0$, then $Q \subset \operatorname{Bs}(\mathcal{L})$.

Proof. From the exact sequence

$$
0 \longrightarrow \mathcal{L}-Q \longrightarrow \mathcal{L} \longrightarrow \mathcal{L}_{\mid Q} \longrightarrow 0
$$

we get $v(\mathcal{L})=v(\mathcal{L}-Q)+v\left(\mathcal{L}_{\mid Q}\right)+1$. If we compare with (1.2) we obtain that

$$
v\left(\mathcal{L}_{\mid Q}\right)=\frac{Q(\mathcal{L}-Q)(\mathcal{L}-K)}{2}-1<0 .
$$

The system $\mathcal{L}_{\mid Q}=\mathcal{L}_{Q}\left((d, d), m_{1}, \ldots, m_{9}\right)$ is equivalent to the planar system $\mathcal{L}_{2}=$ $\mathcal{L}_{2}\left(2 d-m_{1},\left(d-m_{1}\right)^{2}, m_{2}, \ldots, m_{9}\right)$ (see [10]). Therefore in order to prove the claim it is enough to prove that the system $\mathcal{L}_{2}$ is non-special or, since we assume that the Harbourne-Hirschowitz Conjecture holds for 10 multiple points, to prove that $\mathcal{L}_{2}$ is not $(-1)$-special. Let us compare the multiplicity $d-m_{1}$ to the $m_{i}$ 's.

If $d-m_{1} \geq m_{3}$, the system $\mathcal{L}_{2}$ is Cremona-stable (since $2 d-m_{1} \geq 2\left(d-m_{1}\right)+m_{2}$ ), and hence it is not special.

If $d-m_{1}<m_{4}$ and $\mathcal{L}_{2}$ is not Cremona-stable, then $2 d-m_{1}<m_{2}+m_{3}+m_{4}$, which is not possible since we are assuming that $\mathcal{L}$ is in standard form.

Finally, if $m_{4} \leq d-m_{1}<m_{3}$ and $\mathcal{L}_{2}$ is not Cremona-stable, then $d \leq m_{2}+m_{3}-1$. If we write $d=m_{2}+m_{3}-t$, with $t \geq 1$, then

$$
\mathcal{L}_{2}=\mathcal{L}_{2}\left(2 m_{2}+2 m_{3}-m_{1}-2 t, m_{2}, m_{3},\left(m_{2}+m_{3}-m_{1}-t\right)^{2}, m_{4}, \ldots, m_{9}\right) .
$$

Performing a Cremona transformation with the first three points we obtain a stable system (the degree equals the sum of the highest multiplicities). 
Now we wonder: which system of quadrics may be contained in the base locus of a given linear system? The answer is given by the following.

Lemma 4.3. If $\left|\sum_{i=1}^{n} r_{i} Q_{i}\right|$ is contained in the base locus of a linear system, then the $Q_{i}$ must share 8 points, i.e. $Q_{i} \in \mathcal{L}_{3}\left(2,1^{8}\right)$ for each $i$.

Proof. Consider any two of the given quadrics, say $Q_{1}, Q_{2}$, which share $s \leq 8$ points. Since $\left|Q_{1}+Q_{2}\right|=\mathcal{L}_{3}\left(4,2^{s}, 1^{18-2 s}\right)$ has virtual dimension $16-2 s$, the system moves unless $s=8$. Now consider any three quadrics, say $Q_{1}, Q_{2}, Q_{3}$. If they have less than 8 common points, then $\left|Q_{1}+Q_{2}+Q_{3}\right|=\mathcal{L}_{3}\left(6,3^{7}, 2^{3}\right)$ since, by the preceding observation, each pair must share 8 points. But this system has virtual dimension 1 , which is not possible. If four or more quadrics share less than 8 points, then there exist three of them which do not share 8 points, and this is not possible as proved before.

On the other hand, let us prove that if $s=8$, then the system $\mathcal{L}=\left|\sum_{i=1}^{n} r_{i} Q_{i}\right|$ cannot move. We can write $\mathcal{L}=\mathcal{L}_{3}\left(2 r, r^{8}, r_{1}, \ldots, r_{n}\right)$, where $r=\sum_{i=1}^{n} r_{i}$. From the exact sequence of $Q_{1}$ we get

$$
0 \longrightarrow \mathcal{L}-Q_{1} \longrightarrow \mathcal{L} \longrightarrow \mathcal{L}_{\mid Q_{1}} \longrightarrow 0 .
$$

Let us consider the restricted system $\mathcal{L}_{\mid Q_{1}}=\mathcal{L}_{Q}\left((2 r, 2 r), r^{8}, r_{1}\right)$. By [10] this is equivalent to the planar system $\mathcal{L}_{2}\left(3 r, r^{9}, r_{1}\right)$, which is empty since $\mathcal{L}_{2}\left(3 r, r^{9}\right)$ is the fixed cubic $\mathcal{L}_{2}\left(3,1^{9}\right)$ counted $r$ times. Therefore also $\mathcal{L}_{\mid Q_{1}}=\emptyset$, which implies that $Q_{1}$ is contained in the base locus of $\mathcal{L}$. This also implies that $r_{1} Q_{1}$ is contained in the base locus of $\mathcal{L}$, and the same holds for $r_{i} Q_{i}$. Therefore $\mathcal{L}=\sum_{i=1}^{n} r_{i} Q_{i}$.

In order to conclude the part of the procedure concerning quadrics we need the following.

Lemma 4.4. Let us suppose that the Harbourne-Hirschowitz Conjecture holds for linear systems on $\mathbb{P}^{2}$ with 10 points. If $\mathcal{L}=\mathcal{L}_{3}\left(d, m_{1}, \ldots, m_{r}\right)$ is in standard form and $Q(\mathcal{L}-Q)(\mathcal{L}-K)<0$, where $Q$ is the quadric through the first 9 points, then $\mathcal{L}-Q$ is still in standard form.

Proof. By hypothesis $2 d \geq \sum_{i=1}^{4} m_{i}$; moreover the degree of $\mathcal{L}-Q$ is $d-2$ while the first 9 multiplicities are $m_{i}-1$. Therefore $\mathcal{L}-Q$ is in standard form unless $m_{10}>m_{4}-1$. But this can happen only if $m_{i}=m$ for $i=4, \ldots, 10$. In this case $\mathcal{L}$ would contain all the quadrics through $p_{1}, p_{2}, p_{3}$ and 6 of the points $p_{4}, \ldots, p_{10}$, but by Lemma 4.3 this is not possible.

Therefore, assuming that the Harbourne-Hirschowitz Conjecture holds for up to 10 points, we can proceed by computing $Q(\mathcal{L}-Q)(\mathcal{L}-K)$. If it is negative we can remove the quadric $Q$ and consider the system $\mathcal{L}-Q$, which is still in standard form. We reorder the multiplicities if necessary, and we keep removing the quadric as long as $Q(\mathcal{L}-Q)(\mathcal{L}-K)<0$.

Let us consider part (ii) of Conjecture 4.1. We are going to prove that, in fact, such a system $\mathcal{L}$ is special.

Proposition 4.5. Let $\mathcal{L}=\mathcal{L}_{3}\left(d, m_{1}, \ldots, m_{r}\right)$ be a non-empty linear system and let $l$ be the line through $p_{1}, p_{2}$. If $\mathcal{L} l=-t \leq-1$, then $h^{2}\left(\mathcal{L} \otimes \mathcal{I}_{t l}\right)=0$.

Proof. Let $Z=Z^{\prime}+Z^{\prime \prime}$, where $Z^{\prime}=m_{1} p_{1}+m_{2} p_{2}$ and $Z^{\prime \prime}=m_{3} p_{3}+\ldots+m_{r} p_{r}$, and let $\mathcal{L}^{\prime}=\mathcal{O}_{\mathbb{P}^{3}}(d) \otimes \mathcal{I}_{Z^{\prime}}$. The tensor product of the defining sequence of $Z^{\prime \prime}$ with 
$\mathcal{L}^{\prime} \otimes \mathcal{I}_{t l}$ gives

$$
0 \longrightarrow \mathcal{L} \otimes \mathcal{I}_{t l} \longrightarrow \mathcal{L}^{\prime} \otimes \mathcal{I}_{t l} \longrightarrow \mathcal{O}_{Z^{\prime \prime}} \longrightarrow 0 .
$$

Since $h^{i}\left(\mathcal{O}_{Z^{\prime \prime}}\right)=0$ for $i \geq 1$, it is enough to prove that $h^{2}\left(\mathcal{L}^{\prime} \otimes \mathcal{I}_{t l}\right)=0$.

Let us take a plane $V \in \mathcal{L}\left(1,1^{2}\right)$ and denote by $W$ the corresponding element of $\mathcal{L}\left(1,1^{2}\right) \otimes \mathcal{I}_{l}$. From the defining sequence of $W$ we obtain

$$
0 \longrightarrow\left(\mathcal{L}^{\prime}-V\right) \otimes \mathcal{I}_{(t-1) l} \longrightarrow \mathcal{L}^{\prime} \otimes \mathcal{I}_{t l} \longrightarrow\left(\mathcal{L}^{\prime} \otimes \mathcal{I}_{t l}\right)_{\mid W} \longrightarrow 0 .
$$

Observe that $h^{2}\left(\left(\mathcal{L}^{\prime} \otimes \mathcal{I}_{t l}\right)_{\mid W}\right)=h^{2}\left(\mathcal{O}_{\mathbb{P}^{2}}(d-t) \otimes \mathcal{I}_{Z^{\prime}}\right)=0$, since $t=m_{1}+m_{2}-d \leq d$ (otherwise $m_{1}>d$, and the system would be empty). This means that

$$
h^{2}\left(\left(\mathcal{L}^{\prime}-V\right) \otimes \mathcal{I}_{(t-1) l}\right) \geq h^{2}\left(\mathcal{L}^{\prime} \otimes \mathcal{I}_{t l}\right),
$$

so we can proceed by induction on $t$ until we obtain the system

$$
\mathcal{L}_{3}\left(d-t, m_{1}-t, m_{2}-t\right)
$$

whose $h^{2}$ vanishes.

An easy consequence of Corollary 3.3 and Proposition 4.5 is the following.

Corollary 4.6. Let $\mathcal{L}$ and $l$ be as before. If $\mathcal{L} l=-t \leq-2$, then $\mathcal{L}$ is special and $\operatorname{dim} \mathcal{L}-v(\mathcal{L}) \geq\left(\begin{array}{c}t+1 \\ 3\end{array}\right)$.

Observe that if there exists $C_{i}$ with $i \geq 2$, such that $\mathcal{L} C_{i}=-t_{i} \leq-2$, then by the corollary above $\mathcal{L}$ is special. It may happen that $h^{i}\left(\mathcal{L} \otimes \mathcal{I}_{\Gamma}\right)>0$ for $i=1,2$, where $\Gamma=\sum t_{i} C_{i}$. This means that we are not able to compute the speciality of $\mathcal{L}$ (see Example [7.6). For a system in standard form, we conjecture that $h^{2}\left(\mathcal{L} \otimes \mathcal{I}_{\Gamma}\right)=0$ (this has been proved up to eight multiple points in 4]). Moreover, by assuming Conjecture 4.1 after removing the quadrics in $\operatorname{Bs}(\mathcal{L})$, we have that $h^{1}\left(\mathcal{L}^{\prime} \otimes \mathcal{I}_{\Gamma}\right)=0$, where $\mathcal{L}^{\prime}$ is the residual system. This allows us to give the procedure shown in Table 1 for the evaluation of $\operatorname{dim} \mathcal{L}$.

\section{TABLE 1.}

$$
\begin{aligned}
& \text { While } \mathcal{L} \text { is not in standard form } \\
& \text { put } \mathcal{L}:=\operatorname{Cr}(\mathcal{L}) \text {. } \\
& \quad \text { If } m_{i}=-\alpha_{i}<0 \text { then put } \mathcal{L}:=\mathcal{L}-\alpha_{i} E_{i} \\
& \text { While } Q(\mathcal{L}-Q)(\mathcal{L}-K)<0 \\
& \text { put } \mathcal{L}:=\mathcal{L}-Q . \\
& \text { Put } t_{i j}:=m_{i}+m_{j}-d . \quad \text { Return } \operatorname{dim} \mathcal{L}=v(\mathcal{L})+\sum_{t_{i j} \geq 2}\left(\begin{array}{c}
t_{i j}+1 \\
3
\end{array}\right)
\end{aligned}
$$

\section{Motivations}

In 4. Conjecture 4.1] has been proved for systems $\mathcal{L}_{3}\left(d, m_{1}, \ldots, m_{r}\right)$ with $r \leq 8$. With the help of a procedure written in the programming language of the computer algebra system Singular [6], we verified the conjecture for systems $\mathcal{L}_{3}\left(d, m^{r}\right)$ with $m \leq 7$ and $r \leq 20$. 
A consequence of the conjecture is that the only curves $\ell$ which can give speciality are the images of the line $\ell_{3}\left(1,1^{2}\right)$ by a finite set of Cremona transformations. In one direction, observe that if there exists a curve $\ell$ which is the image of $\ell_{3}\left(1,1^{2}\right)$ by a finite set of Cremona transformations and such that $\mathcal{L} \ell=-t \leq-2$, then $\mathcal{L}$ is special. In fact, performing back Cremona, we get a system $\mathcal{L}^{\prime}$ such that $\mathcal{L}^{\prime} \ell_{3}\left(1,1^{2}\right)=-t$, since the intersection product is invariant under Cremona. Therefore, by Proposition 4.5. since cohomology groups are invariant under birational transformations, $h^{2}\left(\mathcal{L} \otimes \mathcal{I}_{t \ell}\right)=0$, and $\mathcal{L}$ is special. On the other hand, for other classes of curves one can have the following problem.

Example 5.1. Let $\ell=\ell_{3}\left(4,1^{8}\right)$ be the system of quartics through 8 fixed points and suppose that $\mathcal{L} \ell=-t \leq-2$. The intersection product is $4 d-\sum_{i=1}^{8} m_{i} \leq-2$, and hence necessarily $2 d-\sum_{i=1}^{4} m_{i} \leq-1$, which implies that $\mathcal{L}$ is not in standard form. Let us perform a Cremona transformation involving the first 4 points. Since the intersection product is invariant under this transformation, $\mathcal{L}^{\prime} \ell^{\prime}=\mathcal{L} \ell=-t$, where $\mathcal{L}^{\prime}=\operatorname{Cr}(\mathcal{L})$ and $\ell^{\prime}=\operatorname{Cr}(\ell)$. Moreover the degree $d^{\prime}$ of $\mathcal{L}^{\prime}$ is strictly smaller than $d$ and, by Proposition [3.9] $\ell^{\prime}=\ell$. Arguing as before we can say that $\mathcal{L}^{\prime}$ is not in standard form, and we can perform another transformation. Iterating this procedure we can transform $\mathcal{L}$ until we get a system having negative degree, and hence we conclude that $\mathcal{L}=\emptyset$. Therefore the quartic cannot give speciality. The fact that $\ell_{3}\left(4,1^{8}\right)$ cannot be obtained from $\ell_{3}\left(1,1^{2}\right)$ by a finite set of Cremona transformations is also an easy consequence of Corollary 3.10, since the invariant $\delta^{2}-\sum 2 \mu_{i}^{2}$ is 0 for the quartic and -3 for the line.

Another consequence of Conjecture 4.1 is that if $\mathcal{L}$ is in standard form, then we cannot find any curve $\ell \neq \ell_{3}\left(1,1^{2}\right)$ which is the image of a line and such that $\mathcal{L} \ell \leq-2$. In order to give a motivation for this we prove the following.

Lemma 5.2. Let us suppose that $\mathcal{L}=\mathcal{L}_{3}\left(d, m_{1}, \ldots, m_{r}\right)$ and $\ell$ is obtained from $\ell_{3}\left(1,1^{2}\right)$ by a finite set of Cremona transformations such that at each step the degree of the curve increases. Then we can write

$$
\mathcal{L} \ell=\beta_{1}\left(2 d-\sum_{i=1}^{4} m_{i}^{(1)}\right)+\cdots+\beta_{a}\left(2 d-\sum_{i=1}^{4} m_{i}^{(a)}\right)+\left(d-m_{h}-m_{k}\right),
$$

where $\beta_{j} \geq 1, m_{i}^{(j)}$ are chosen between $m_{1}, \ldots, m_{r}$ and $h, k \geq 5$.

Proof. We argue by induction on the number of Cremona transformations necessary to obtain $\ell$ from the line $\ell_{3}\left(1,1^{2}\right)$. First of all, after one transformation the image of the line is the rational normal cubic $\ell_{3}\left(3,1^{6}\right)$, having intersection product with $\mathcal{L}$ equal to $3 d-\sum_{i=1}^{6} m_{i}=\left(2 d-\sum_{i=1}^{4} m_{i}\right)+\left(d-m_{5}-m_{6}\right)$. Now we assume that the formula is true for $\ell=\ell_{3}\left(\delta, \mu_{1}, \ldots, \mu_{s}\right)$, and we prove it for the curve $\ell^{\prime}=\operatorname{Cr}(\ell)=\ell_{3}\left(\delta^{\prime}, \mu_{1}^{\prime}, \ldots, \mu_{s}^{\prime}\right)$ obtained from $\ell$ performing one more Cremona transformation increasing the degree. We can suppose that the transformation is based on the first 4 points. By formula (3.5), $\delta^{\prime}=\delta+2 \gamma$ and $\mu_{i}^{\prime}=\mu_{i}+\gamma$ for $i=1, \ldots, 4$, where $\gamma=\delta-\sum_{i=1}^{4} \mu_{i}>0$, and $\mu_{i}^{\prime}=\mu_{i}$ for $i \geq 5$. Therefore $\mathcal{L} \ell^{\prime}-\mathcal{L} \ell=\gamma\left(2 d-\sum_{i=1}^{4} m_{i}\right)$, which proves the claim. 
Corollary 5.3. If $\mathcal{L}$ is not empty and in standard form and $\ell$ is obtained from $\ell_{3}\left(1,1^{2}\right)$ by a finite set of Cremona transformations such that at each step the degree of the curve increases, then $\mathcal{L} \ell \geq 0$.

Proof. Since $\mathcal{L}$ is in standard form, $2 d \geq \sum m_{i}^{(j)}$. Moreover $d-m_{h}-m_{k} \geq 0$, since otherwise $2 d<m_{1}+m_{2}+m_{h}+m_{k}$. Hence all the terms on the right side of (5.1) are non-negative.

Therefore, if we assume that the following conjecture holds, we have that a system in standard form cannot have a negative intersection product with the image of a line.

Conjecture 5.4. Let $\ell=\ell_{3}\left(\delta, \mu_{1}, \ldots, \mu_{r}\right)$ be a curve that can be obtained from the line $\ell_{3}\left(1,1^{2}\right)$ by a finite set of Cremona transformations. Then $\ell$ can be obtained by a finite set of Cremona transformations such that at each step the degree of the curve increases.

\section{Homogeneous linear systems}

In this section we study special homogeneous systems $\mathcal{L}=\mathcal{L}_{3}\left(d, m^{r}\right)$.

Proposition 6.1. The system $\mathcal{L}$ is empty for $d \leq 2 m-1$ and $r \geq 8$.

Proof. It is enough to show that the system $\mathcal{L}_{3}\left(2 m-1, m^{8}\right)$ is empty. We first prove by induction that performing Cremona on the first four points and the last four alternatively, at the $i$-th step we get the system

$$
\mathcal{L}^{i}=\mathcal{L}_{3}\left(2 m-2 i^{2}-1,\left(m-i^{2}+i\right)^{4},\left(m-i^{2}-i\right)^{4}\right) .
$$

The basis of induction holds since applying Cremona to the starting system we get $\mathcal{L}^{1}=\mathcal{L}_{3}\left(2 m-3,(m-2)^{4}, m^{4}\right)$. If we now perform Cremona to $\mathcal{L}^{i}$ taking the first four points we have that $k=2\left(2 m-2 i^{2}-1\right)-4\left(m-i^{2}+i\right)=-4 i-2$ and hence the transform system is

$$
\operatorname{Cr}\left(\mathcal{L}^{i}\right)=\mathcal{L}_{3}\left(2 m-2(i+1)^{2}-1,\left(m-(i+1)^{2}-(i+1)\right)^{4},\left(m-i^{2}-i\right)^{4}\right) .
$$

The multiplicity $m-i^{2}-i$ can be written as $m-(i+1)^{2}+(i+1)$, and hence, reordering the points, we get that $\operatorname{Cr}\left(\mathcal{L}^{i}\right)=\mathcal{L}^{i+1}$, which gives the inductive step.

We keep performing these transformations until $m-i^{2}-i \leq 0$. At this step we have that $2 m-2 i^{2}-1<m-i^{2}+i$, and hence the system $\mathcal{L}^{i}$ is empty since it has some multiplicity bigger than the degree.

By assuming Conjecture 4.1 and the Harbourne-Hirschowitz Conjecture for linear systems on $\mathbb{P}^{2}$ with 10 points, we can prove the following.

Proposition 6.2. If $d \geq 2 m$ the system $\mathcal{L}=\mathcal{L}_{3}\left(d, m^{r}\right)$ is special if and only if $r=9$ and $2 m \leq d<\left[-1+\frac{3}{2} \sqrt{2 m^{2}+2 m}\right]$.

Proof. Our assumption on the degree implies that $\mathcal{L}$ is in standard form. According to Conjecture 4.1, the system $\mathcal{L}$ is special if and only if there exists a quadric $Q \in \mathcal{L}_{3}\left(2,1^{9}\right)$ such that $Q(\mathcal{L}-Q)(\mathcal{L}-K)<0$, or if there exists a line $\ell=\ell_{3}\left(1,1^{2}\right)$ such that $\ell \mathcal{L} \leq-2$. But under our hypothesis on the degree, a line through 2 fixed points has non-negative intersection product with $\mathcal{L}$, and hence $\mathcal{L}$ is special if and only if $Q(\mathcal{L}-Q)(\mathcal{L}-K)<0$. But we can write $Q(\mathcal{L}-Q)(\mathcal{L}-K)=2(d-2)(d+4)-$ $9(m-1)(m+2)$, which is negative if and only if $2 m \leq d<\left[-1+\frac{3}{2} \sqrt{2 m^{2}+2 m}\right]$. We finish the proof by showing that if $\mathcal{L}$ is special, then the number $r$ of fixed 
points cannot be bigger than 9 . If we suppose by contradiction that $r \geq 10$, then $Q_{i}\left(\mathcal{L}-Q_{i}\right)(\mathcal{L}-K)<0$, where $Q_{i}$ is the quadric through all the first 10 points but $p_{i}$. By Lemma $4.2 \mathcal{L}$ must contain the system of quadrics $\sum_{i=1}^{10} Q_{i}$, and this is not possible because of Lemma 4.3 .

We can conclude that if the system $\mathcal{L}$ has more than 9 fixed points (or exactly 8 points), then it is not special. If it has 9 fixed points, it is special if and only if $2 m \leq d<\left[-1+\frac{3}{2} \sqrt{2 m^{2}+2 m}\right]$. If $r \leq 7$ and $d \geq 2 m$, the system cannot be special. Finally, if $r \leq 7$ and $d \leq 2 m-1$, we have to follow the procedure of Table 1.

\section{EXAMPLES}

We end the paper by presenting many examples of special systems and computing their dimension following the procedure of Table 1. We remark that for each example the dimension we found in this way agrees with the dimension computed with the help of a Singular program.

Notation 7.1. We will use the symbols $\stackrel{(\text { i) }}{\sim}, \stackrel{(i i)}{\rightsquigarrow}$ and $\stackrel{(\text { iii) }}{\rightsquigarrow}$ to signify that we are applying the first, the second and the third step of the procedure (in (i) we are applying a Cremona transformation, in (ii) we are removing a plane and in (iii) we are removing a quadric).

Example 7.2. Consider the system $\mathcal{L}:=\mathcal{L}_{3}\left(7,4^{6}\right)$ with $v(\mathcal{L})=-1$. Following the procedure of Table 1 we can apply four Cremona transformations:

$$
\mathcal{L}_{3}\left(7,4^{6}\right) \stackrel{(\mathrm{i})}{\rightsquigarrow} \mathcal{L}_{3}\left(5,4^{2}, 2^{4}\right) \stackrel{(\mathrm{i})}{\rightsquigarrow} \mathcal{L}_{3}\left(3,2^{4}\right) \stackrel{(\mathrm{i})}{\rightsquigarrow} \mathcal{L}_{3}(1),
$$

so that $\operatorname{dim} \mathcal{L}=3$. Observe that since $\mathcal{L}_{3}\left(7,4^{6}\right) \ell_{3}\left(3,1^{6}\right)=-3$, the starting system contains the rational normal curve. After the first transformation $\ell_{3}\left(3,1^{6}\right) \rightsquigarrow$ $\ell_{3}\left(1,1^{2}\right)$ and, in fact, $\mathcal{L}_{3}\left(5,4^{2}, 2^{4}\right) \ell_{3}\left(1,1^{2}\right)=-3$.

Example 7.3. Consider the system $\mathcal{L}:=\mathcal{L}_{3}\left(12,7^{6}\right)$ with $v(\mathcal{L})=-50$. As before we can apply the following Cremona transformations:

$$
\mathcal{L}_{3}\left(12,7^{6}\right) \stackrel{(\text { i })}{\rightsquigarrow} \mathcal{L}_{3}\left(8,7^{2}, 3^{4}\right) \stackrel{(\text { i) }}{\rightsquigarrow} \mathcal{L}_{3}\left(4,3^{4},-1^{2}\right) \stackrel{(\text { ii) }}{\rightsquigarrow} \mathcal{L}_{3}\left(4,3^{4}\right) \stackrel{(\text { i) }}{\rightsquigarrow} \mathcal{L}_{3}\left(0,-1^{4}\right) \stackrel{(\text { ii) }}{\rightsquigarrow} \mathcal{L}_{3}(0),
$$

so that $\operatorname{dim} \mathcal{L}=0$. From the procedure we may deduce that $\mathcal{L}$ is given by the union of six surfaces of type $\mathcal{L}_{3}\left(2,2,1^{5}\right)$ (each surface can be obtained from a plane $\mathcal{L}_{3}\left(1,1^{3}\right)$ applying one Cremona transformation).

In these two first examples, by performing some Cremona transformations and removing fixed planes we get a system in standard form which is not special. Let us give two examples where the system in standard form is still special.

Example 7.4. Consider the system $\mathcal{L}:=\mathcal{L}_{3}\left(10,6^{5}\right)$ with $v(\mathcal{L})=5$. Apply the Cremona transformation:

$$
\mathcal{L}_{3}\left(10,6^{5}\right) \stackrel{(\mathrm{i})}{\rightsquigarrow} \mathcal{L}_{3}\left(6,6,2^{4}\right) .
$$

The last system is in standard form and has virtual dimension 11 while its dimension is 15 , as expected by our conjecture. In fact each of the four lines $l_{1 j}, j=2, \ldots, 5$, has intersection product -2 with the system. 
Example 7.5. Consider the system in standard form $\mathcal{L}:=\mathcal{L}_{3}\left(16,11,7^{8}\right)$ with $v(\mathcal{L})=10$. Since $Q(\mathcal{L}-Q)(\mathcal{L}-K)=-2$ following the procedure, we may remove the quadric from the base locus of $\mathcal{L}$ :

$$
\mathcal{L}_{3}\left(16,11,7^{8}\right) \stackrel{(\mathrm{iii})}{\rightsquigarrow} \mathcal{L}_{3}\left(14,10,6^{8}\right) .
$$

The virtual dimension of the last system is 11 , and its dimension is 19 (each of the 8 lines $l_{1 j}, j=2, \ldots, 9$, has intersection product -2 with the system).

We remark that in the two examples above, when the linear system is in standard form and does not contain any quadric, then its speciality is given exactly by the sum of the binomials $\left(\begin{array}{c}t_{i j}+1 \\ 3\end{array}\right)$, where $-t_{i j}$ is the intersection product of the line $l_{i j}$ with the system. In the following example we show that if a linear system $\mathcal{L}$ is not in standard form, then its speciality can be strictly smaller than the sum of these binomials, or equivalently $h^{2}\left(\mathcal{L} \otimes \mathcal{I}_{\Gamma}\right) \neq 0$ (where $\Gamma$ is the 1 -dimensional scheme of the multiple lines).

Example 7.6. The system $\mathcal{L}=\mathcal{L}_{3}\left(3,3^{3}\right)$ with $v(\mathcal{L})=-11$ is not in standard form, hence

$$
\mathcal{L}_{3}\left(3,3^{3}\right) \stackrel{(\mathrm{i})}{\rightsquigarrow} \mathcal{L}_{3}(0,-3) \stackrel{(\mathrm{ii})}{\rightsquigarrow} \mathcal{L}_{3}(0) .
$$

This implies that $\operatorname{dim} \mathcal{L}=0$, since it is three times the plane $\mathcal{L}_{3}\left(1,1^{3}\right)$. For each line $l_{i j}$ through two of the three points we have $\mathcal{L} l_{i j}=-3$. Let $\Gamma=3\left(l_{12}+l_{13}+l_{23}\right)$. By Corollary $3.3 \operatorname{dim} \mathcal{L}-v(\mathcal{L}) \geq 12-h^{2}\left(\mathcal{L} \otimes \mathcal{I}_{\Gamma}\right)$, which implies that $h^{2}\left(\mathcal{L} \otimes \mathcal{I}_{\Gamma}\right) \geq 1$.

In the next example we give a way to construct a class of systems whose speciality is due to multiple quadrics in the fixed locus.

Example 7.7. Let $r_{1}, \ldots, r_{n}$ be positive integers and let $r=\sum r_{i}$. As we proved in Lemma 4.3. the system $\mathcal{L}=\mathcal{L}_{3}\left(2 r, r^{8}, r_{1}, \ldots, r_{n}\right)$ has dimension 0 , while its virtual dimension is $\sum\left(r_{i}-\left(\begin{array}{c}r_{i}+2 \\ 3\end{array}\right)\right) \leq 0$. Moreover this sum is 0 if and only if each $r_{i}=1$, otherwise the system is special.

\section{REFERENCES}

[1] Ciro Ciliberto, Geometric aspects of polynomial interpolation in more variables and of Waring's problem, European Congress of Mathematics, Vol. I (Barcelona, 2000), 289-316, Progr. Math., 201, Birkhäuser, Basel, 2001. MR,1905326 (2003i:14058)

[2] Ciro Ciliberto and Rick Miranda, Degenerations of planar linear systems, J. Reine Angew. Math. 501 (1998), 191-220. MR1637857 (2000m:14005)

[3] — Linear systems of plane curves with base points of equal multiplicity, Trans. Am. Math. Soc. 352 (2000), no. 9, 4037-4050 (English). MR.1637062 (2000m:14006)

[4] Cindy De Volder and Antonio Laface, On linear systems of $\mathbb{P}^{3}$ through multiple points, preprint.

[5] William Fulton, Intersection theory, Second edition, Springer, Berlin, 1998. MR 1644323 (99d:14003)

[6] G.-M. Greuel, G. Pfister, and H. Schönemann, Singular 2.0, A Computer Algebra System for Polynomial Computations, Centre for Computer Algebra, University of Kaiserslautern, 2001, http://www. singular.uni-kl.de.

[7] Brian Harbourne, The geometry of rational surfaces and Hilbert function of points in the plane, Can. Math. Soc. Conf. Proc. 6 (1986), 95-111. MR0846019 (87k:14041)

[8] Robin Hartshorne, Algebraic Geometry, Springer, New York, 1977. MR0463157 (57:3116)

[9] André Hirschowitz, Une conjecture pour la cohomologie des diviseurs sur les surfaces rationnelles génériques, J. Reine Angew. Math. 397 (1989), 208-213. MR0993223 (90g:14021)

[10] Antonio Laface and Luca Ugaglia, A counterexample to a conjecture on linear systems on $\mathbb{P}^{3}$, to appear in Advances in Geometry. MR2071811 (2005c:14008) 
[11] Evain Laurent, La fonction de Hilbert de la réunion de $4^{h}$ gros points génériques de $\mathbf{p}^{2}$ de même multiplicité, J. Algebraic Geom. 8 (1999), no. 4, 787-796. MR.1703614(2000e:13023)

[12] Thierry Mignon, Systèmes de courbes planes à singularités imposées: le cas des multiplicités inférieures ou égales à quatre, J. Pure Appl. Algebra 151 (2000), no. 2, 173-195. MR.1775572 (2001g:14048)

Dipartimento di Matematica, Università degli Studi di Milano, Via Saldini 50, 20100 Milano, Italy

E-mail address: antonio.laface@unimi.it

Current address: Department of Mathematics and Statistics, Queen's University, Kingston, Ontario, Canada K7L 3N6

E-mail address: alaface@mast.queensu.ca

Dipartimento di Matematica, Università degli Studi di Milano, Via Saldini 50, 20100 Milano, Italy

E-mail address: luca.ugaglia@unimi.it

Current address: Via Petrarca 24, 14100 Asti, Italy

E-mail address: luca.ugaglia@gmail.com 